\title{
New Cooperative Effects in Ballistic Deposition of Hard Disks
}

\author{
Jordi Faraudo* \\ Department of Chemistry, Imperial College of Science, Technology and Medicine, \\ Exhibition Road, London SW7 2AY, United Kingdom \\ (Received 7 October 2002; published 20 December 2002)
}

\begin{abstract}
In this Letter, we analyze the problem of monolayer formation onto a flat surface by deposition of hard particles. Our detailed computer simulations of ballistic deposition of hard disks show significant deviations from the classical analytical solution obtained by J. Talbot et al. [Phys. Rev. Lett. 68, 958 (1992)]. These deviations are due to cooperative adsorption induced by particles trapped above the adsorbed layer. We show that not only the adsorption kinetics but also the jamming (saturation) coverage of the surface depends on the volume fraction of the suspension, a prediction which is completely new. These new cooperative effects cannot be neglected even in the case of very diluted suspensions.
\end{abstract}

DOI: 10.1103/PhysRevLett.89.276104

PACS numbers: 68.43.Mn, 82.70.Dd

The physics of particle deposition is a field with many challenging and interesting problems from a theoretical viewpoint and also with a recognized importance in the understanding of many chemical, biological, and industrial processes. These processes include filtration, mineral processing, the fouling of heat exchanger surfaces, and thrombus formation associated with artificial organs, to mention only a few [1]. In this Letter, we are interested in one of such problems, namely, the irreversible formation of monolayers by deposition of large colloidal particles from fluid suspensions onto solid surfaces. In a typical experiment, one considers a diluted suspension of colloidal particles, which sediment due to the effect of gravity $[2,3]$. We are interested in situations in which the density difference between the solvent and the particles is large, so that the effect of diffusion in the motion of the particles can be neglected. In many experimental conditions [2-5] (for example, in the case of latexes, erythrocytes, etc.), the deposited particles are strongly bound to the adsorbing surface, and neither desorption nor surface diffusion are observed. Therefore, the particles in contact with the surface form a layer of irreversibly adsorbed particles in a kinetically frustrated state. Other particles can deposit over this layer by sedimentation, but they are not bound to the substrate and can easily be removed. Hence, only the particles in the layer in contact with the surface can be considered adsorbed. The macroscopic state of the adsorbed layer is typically characterized by the coverage of the surface $\theta$ which is the fraction of the area $A$ occupied by particles. The coverage $\theta$ increases monotonically with time, and the area of the surface available for adsorption decreases due to the hard core interaction between particles (the so-called blocking effect). The adsorption process may continue until no further particles can adsorb onto the surface and a jamming configuration is obtained. Much effort has been devoted to the study of the deposition kinetics as well as the structure of the adsorbed monolayer both from the ex- perimental and theoretical points of view (see Refs. [4,5] for a review).

In order to be more specific, let us consider the classical problem of deposition of spherical particles onto a planar surface. From the theoretical point of view, it is a standard practice to analyze this deposition process by using the so-called correlated sequential adsorption model, also called the ballistic deposition (BD) model [6]. In this model, the actual deposition process is replaced by some simple geometrical rules for the filling of the adsorbing surface. These rules are as follows: (i) the particles are deposited sequentially on the surface, (ii) for each deposition trial, a starting position is chosen randomly over the adsorption plane. The particle then follows a vertical trajectory until it reaches the adsorption plane or contacts a previously deposited particle. In this latter case, it follows the path of steepest descent until it reaches a stable position. (iii) If this position lies on the adsorbing surface, the particle is irreversibly adsorbed. Otherwise, the particle is removed from the system and a new trial is started. At this point, it is important to recall that multilayer formation is not allowed in the BD model: all trapped particles are simply rejected (eliminated from the system) during the adsorption process. This rule is typically justified by assuming that the formation of the adsorbed layer is much more rapid than the growth of layers of trapped particles. Computer simulations show that the saturation coverage (jamming limit coverage) is given by $\theta_{\infty}=0.6105$ [4]. The kinetics of the deposition process and the structure of the adsorbed monolayer have been analyzed extensively by simulations [4]. In addition, it is possible to derive approximate analytical expressions for the process kinetics valid at the limiting situations of low coverages or high coverages [7]. Also, the simplified situation of ballistic deposition of hard disks onto a line has been solved exactly by Talbot et al. [8]. In this case, the saturation coverage has also been obtained analytically $\left(\theta_{\infty}=0.80865\right)$. The success of the BD model in 
explaining experimental data $[2,4]$ has motivated the introduction of many improvements in the model in order to account for fine details neglected in the original BD model $[9,10]$. In the case of BD of hard disks, the effect of hydrodynamic interactions [9] and external fields [10] has been analyzed.

Despite its success, the basic rules of the BD model are questionable. In particular, the rejection rule in the $\mathrm{BD}$ model is not realistic: in an actual experiment, trapped particles are not withdrawn from the system. In fact, these trapped particles can help new incoming particles to be adsorbed. This produces a cooperative effect which is neglected in the classical BD model. In order to illustrate the limitations of the classical BD model, let us consider the situation illustrated in Fig. 1. In order to simplify the geometry, we consider the adsorption of hard disks onto a line. In Fig. 1 (left), we consider a configuration of two adsorbed disks and two free disks (labeled as 1 and 2) which sediment with constant velocity. After a given time, these incoming particles collide with the adsorbed ones. In the classical BD model, both 1 and 2 are rejected from the simulation and the coverage of the line is unchanged. However, one expects that the final configuration of particles is that illustrated in Fig. 1 (right): particle 1 is trapped in a stable mechanical position and particle 2 is adsorbed after rolling over particle 1 . This kind of cooperative effects and other more complex effects (involving more particles) are unavoidable in experimental situations and are ignored by the BD model in its present formulation. Usually, it is assumed that if the bulk concentration of particles is small (as it is in typical experiments), this kind of effects can be neglected and the usual BD is valid. However, it is not clear how these cooperative mechanisms can be suppressed by decreasing the volume fraction. In fact, there is no theoretical study in the literature supporting this strong hypothesis. Our objective in this work is to elucidate under which conditions this hypothesis can be accepted and which kind of deviations from the BD model appear under reasonable conditions. This objective will be accomplished by comparing computer simulations with the known results of the BD model.
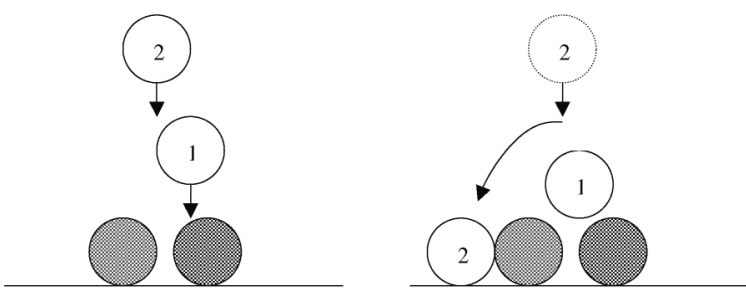

FIG. 1. Example of cooperative effects in adsorption. Left: initial configuration with two adsorbed particles and two incoming particles (labeled as 1 and 2). Right: disk 1 is deposited over the previously adsorbed particles and disk 2 is adsorbed at $z=0$ after rolling over disk 1 .
Detailed computer simulations of the deposition process without the simplifying hypothesis of BD are prohibitive from the computational point of view. In order to perform detailed simulations of deposition using a reasonable amount of computing time, we will consider the deposition of hard disks instead of hard spheres. At this point, we recall that this is a typical procedure in adsorption-deposition studies: in the two dimensional system it is easier to perform simulations and to obtain analytical results $[4,8-10]$. The results obtained in the two dimensional system are very useful to guide the analysis of the much more complicated three dimensional system. Of course, this procedure is reasonable if no new adsorption mechanisms appear in the three dimensional problem, being the 3D and $2 \mathrm{D}$ processes essentially driven by the same mechanisms. We will comment on the validity of our results in the 3D case later on.

Now, let us describe in detail our model and the simulation algorithm. Let $L_{x}$ be the length of the adsorbing surface, $L_{z}$ the maximum height of the adsorption cell, and $N_{B}$ the initial number of disks in the suspension. The initial concentration and volume fraction are given, respectively, by $c_{B}=N_{B} /\left(L_{z} L_{x}\right)$ and $\phi_{B}=\pi R^{2} c_{B}$, where $R$ is the radius of the disks. During the whole adsorption process, no particles are added or subtracted from the system (closed cell conditions). All particles move with the same constant vertical sedimentation velocity $v_{S}$ and interact between them with hard core interactions. Hence, in the suspension there is a vertical flux of particles towards $z=0$ given by $J_{0}=v_{S} c_{B}$. At $z=0$ there is an irreversibly adsorbing surface: when the center of a particle reaches $z=0$, it is immobilized. As time passes, one observes the formation of an adsorbed layer at $z=0$. Also, particles trapped into stable mechanical positions over other deposited particles can be observed. In adsorption processes, it is customary to introduce a dimensionless time defined as $\tau \equiv 2 R J_{0} t \equiv 2 R c_{B} v_{S} t$. The kinetics of the layer appearing at $z=0$ is typically characterized by the coverage defined by

$$
\theta(\tau)=N_{a}(\tau) \frac{2 R}{L_{x}}
$$

where $N_{a}(\tau)$ is the number of particles adsorbed at $z=0$ at time $\tau$. In addition, there are $N_{m}(\tau)$ particles trapped into stable mechanical positions over previously deposited particles.

The simulation algorithm is as follows. First, an initial configuration of nonoverlapping hard disks is generated, with volume fraction $\phi_{B}$. We use dimensionless units, in which distances are measured in units of the particle diameter $\left(x^{\prime}=\frac{x}{2 R}, z^{\prime}=\frac{z}{2 R}\right)$ and time is measured in units of $\tau$. At each time step $\Delta \tau$, each free particle is displaced a distance $\Delta z^{\prime}$ given by

$$
\Delta z^{\prime}=-\frac{v_{S}}{2 R} \Delta t=-\frac{\pi}{4 \phi_{B}} \Delta \tau
$$


After each move of a particle, a test is performed in order to prevent unphysical overlaps with the other particles of the system. If an overlap is found, the currently moving particle is displaced along the center-to-center line until the overlap disappears. Also, a test is performed in order to check if the particle is still free, or is adsorbed or trapped into a position of stable mechanical equilibrium. We have performed simulations for different initial bulk volume fractions in the range $10^{-4}-10^{-1}$. In all simulations, $N_{B}=300$, the size of the adsorbing line was $L_{x} / 2 R=100$ and $\Delta z^{\prime}=0.01$. The desired initial volume fraction is obtained by adjusting the vertical dimension $L_{z} / 2 R$. We have stopped the simulations at $\tau=3$.0. At this time, in most simulations the adsorbing surface has reached the saturation or jammed state (no more particles can be adsorbed at $z=0$ ), so the saturation coverage can be computed. In the remaining simulations, some small gaps with room for only one particle remain. Hence, the saturation coverage can be predicted easily by adding to $N_{a}$ one particle per remaining gap. The time evolution of the coverage, averaged over 10000 simulations, is shown in Fig. 2 for $\phi_{B}=7.854 \times 10^{-4}$ and $\phi_{B}=0.1571$. In contrast to the $\mathrm{BD}$ model, the observed adsorption kinetics does depend on the bulk concentration. The deviations appear at adsorption times $\tau \approx 0.5$. At intermediate values of $\tau$, the coverage obtained in simulations is larger

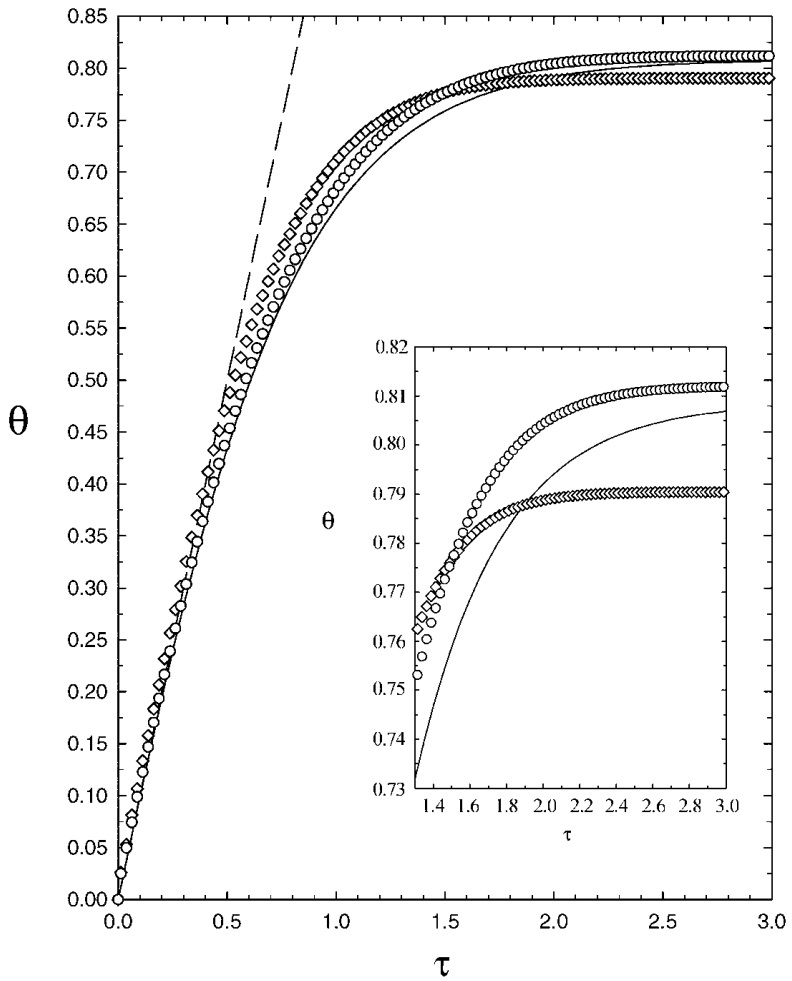

FIG. 2. Adsorption kinetics from simulations with $\phi_{B}=$ $7.854 \times 10^{-4}$ (circles) and $\phi_{B}=0.1571$ (diamonds). The solid line corresponds to the classical BD model and the dashed line to $\theta=\tau$. Inset: Adsorption kinetics near the jamming state. than the coverage predicted by the BD model. This result agrees with the initial expectations based on the mechanism shown in Fig. 1, which predicts an enhancement of adsorption. It is important to note that even for the smallest value analyzed of the volume fraction, deviations from the BD model are still present. The obtained values of the saturation coverage also differ from the BD predictions. We have obtained $\theta_{\infty}=0.8121 \pm 0.0002$ for $\phi_{B}=7.854 \times 10^{-4}$ and $\theta_{\infty}=0.7904 \pm 0.0002$ for $\phi_{B}=$ 0.1571 . In order to investigate the evolution of the saturation coverage with the volume fraction, we have performed additional simulations with different values of $\phi_{B}$. In most of the cases we have performed $10^{3}$ simulations (instead of $10^{4}$ ) for each volume fraction in order to save computer time. The results are shown in Fig. 3 (the error bars represent $95 \%$ confidence intervals). The most striking feature of these results is that $\theta_{\infty}$ is approximately constant up to $\phi_{B} \approx 10^{-2}$ with a saturation coverage slightly larger than the BD predictions. For larger volume fractions, the saturation coverage decreases as the bulk volume fraction increases. All these differences between the BD model and the simulations can be attributed only to the interaction of new incoming particles with particles deposited at layers above $z=0$. Hence, it seems convenient to monitor the growth of multilayers during the simulations. Nearly all the particles deposited in layers over $z=0$ can be found on the second and third

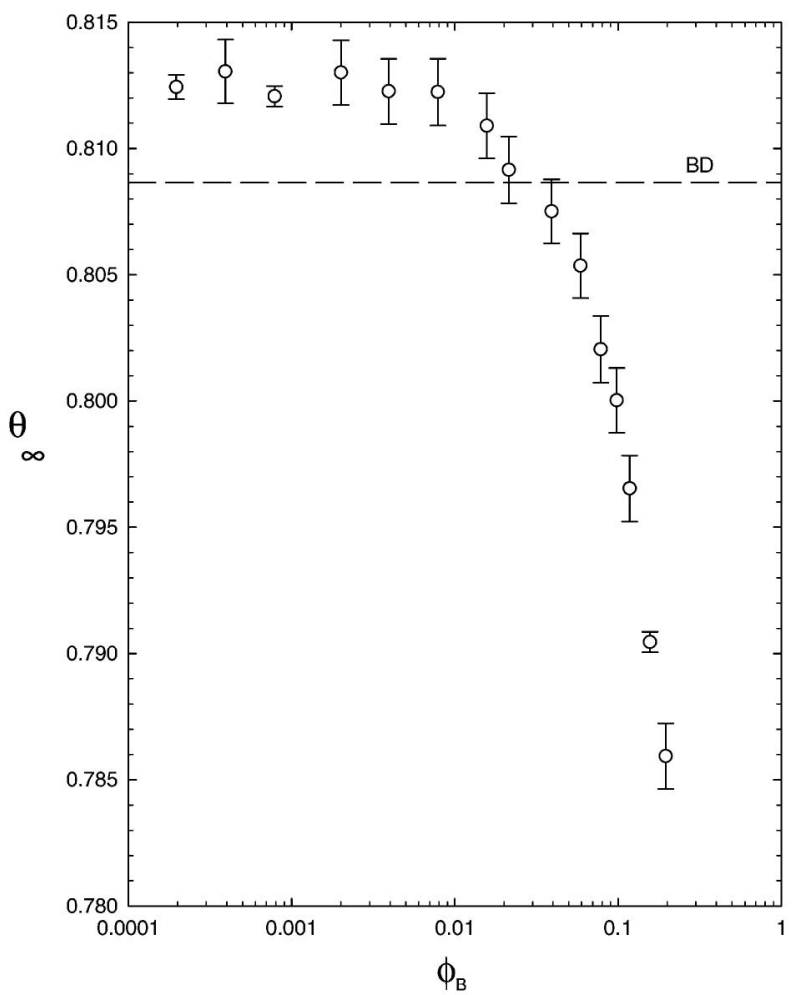

FIG. 3. Saturation coverage observed in simulations as a function of the initial volume fraction. The dashed line corresponds to the $\mathrm{BD}$ model saturation coverage. 


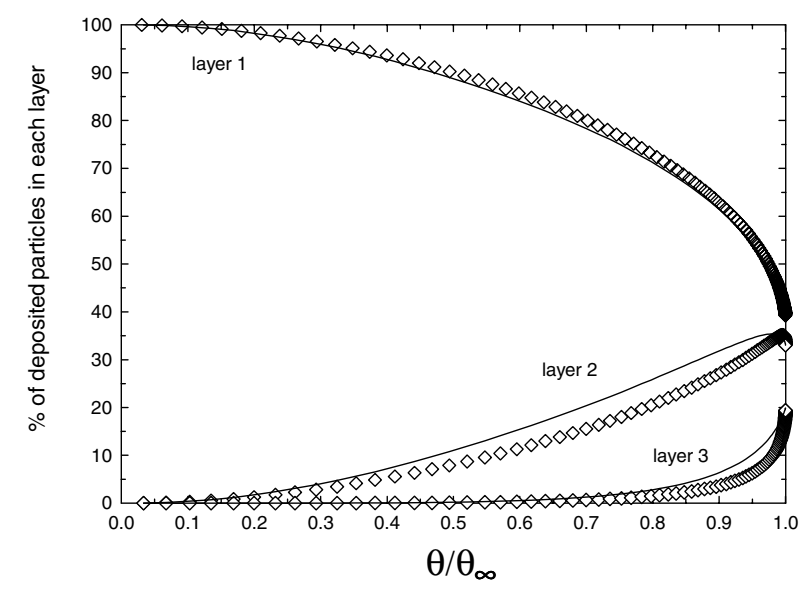

FIG. 4. Fraction of particles deposited in layers 1, 2, and 3 as a function of the relative coverage of the surface. The diamonds correspond to simulations with $\phi_{B}=7.854 \times 10^{-4}$ and the lines correspond to simulations with $\phi_{B}=0.1571$.

layers (particles over the third layer were rarely observed except at stages very close to saturation). We show in Fig. 4 the fraction of all the deposited particles that can be found in the first layer $(z=0)$, the second layer (particles trapped onto particles adsorbed at $z=0$ ), and third layer (particles trapped onto particles at the second layer). From this figure it is clear that the number of particles at secondary layers is small only at the initial stage of the adsorption process. The presence of particles deposited in secondary layers is clear at intermediate coverages, and as the first layer approaches its saturation coverage, the number of particles depositing in multilayers increases dramatically. It is important to note that the fraction of particles deposited in secondary layers does not strongly differ between the cases $\phi_{B}=7.854 \times 10^{-4}$ and $\phi_{B}=$ 0.1571 . This is consistent with the fact that the initial dilution of the suspension does not prevent the mechanism of secondary layer formation. Hence, the cooperative effects induced by these trapped particles cannot be neglected even for very diluted suspensions.

In conclusion, we have shown for the first time that the formation of secondary layers cannot be neglected in ballistic deposition/adsorption studies. We have found that both the kinetics of adsorption and the saturation coverage depend on the volume fraction even for very diluted suspensions. This dependence, absent in the classical BD model, is a consequence of the interaction between incoming bulk particles and particles deposited at layers above the adsorbed layer $(z=0)$. This result has important implications in adsorption/deposition studies, where it is usually assumed that the value of the volume fraction is not an important factor determining adsorption kinetics and jamming coverage provided that it is small. At this point, the obvious question is if this kind of effects, predicted for the 2D system, are observable in actual 3D systems. In a recent paper by Carl et al. [2], experimental results for latexes and erythrocytes are compared with BD predictions and Brownian dynamics simulations. In these simulations, the trapped particles are not removed so multilayer formation is possible. In Fig. 4 of this reference (corresponding to the heavier particles analyzed) a deviation from BD is observed both from experiments and simulations. Qualitatively, the observed behavior is very close to our Fig. 2. Hence, it is very reasonable to infer that the cooperative effects described in the present paper are responsible for the differences observed between experiments (or simulations) and the BD model. Of course, more detailed studies in $3 \mathrm{D}$ models are needed to confirm this hypothesis. But from the present work it seems clear that in any detailed deposition model, the effect of multilayer formation cannot be neglected as is done in classical models. Another very interesting prediction arising for the present work is the dependence of the saturation coverage on the volume fraction. We encourage experimentalists to undertake the necessary experiments to check if this effect is indeed observed in real systems.

The author acknowledges financial support from the Spanish government (MCyT) under Grant No. BFM20000351-C03-01 and from the Generalitat of Catalonia (Direcció general de recerca) under Grant No. 2001-SGR00186. Useful comments by B. Senger, R. DominguezCascante, and J. Bafaluy are also acknowledged.

*On leave from Departament de Física, Universitat Autònoma de Barcelona, E-08193 Bellaterra, Barcelona, Spain.

Electronic adress: jordi.faraudo@uab.es

[1] M. Elimelech, J. Gregory, X. Jia, and R. Williams, Particle Deposition and Aggregation (ButterworthHeinemann Ltd., Oxford, 1995), 1st ed.

[2] P. Carl, M. Schmittbuhl, P. Schaaf, J.-F. Stoltz, J.-C. Voegel, and B. Senger, Physica (Amsterdam) 298A, 198 (2001).

[3] P. Wojtaszczyk, E. K. Mann, B. Senger, J. C. Voegel, and P. Schaaf, J. Chem. Phys. 103, 8285 (1995).

[4] P. Schaaf et al., Ann. Phys. (Paris) 23, 1 (1998); B. Senger et al., Colloids Surf. A 165, 255 (2000).

[5] Z. Adamczyk et al., Adv. Colloid Interface Sci. 48, 151 (1994).

[6] A. P. Thompson and E. D. Glandt, Phys. Rev. A 46, 4639 (1992); R. Jullien and P. Meakin, J. Phys. A 25, L189 (1992).

[7] H. S. Choi, J. Talbot, G. Tarjus, and P. Viot, J. Chem. Phys. 99, 9296 (1993).

[8] J. Talbot and S. M. Ricci, Phys. Rev. Lett. 68, 958 (1992).

[9] I. Pagonabarraga and M. Rubí, Phys. Rev. Lett. 73, 114 (1994).

[10] I. Pagonabarraga, J. Bafaluy, and M. Rubí, Phys. Rev. Lett. 75, 461 (1995). 